\title{
Improved Correlator Peak Selection for GNSS Receivers in Urban Canyons
}

\author{
Peng Xie and Mark G. Petovello \\ (Position, Location And Navigation (PLAN) Group, Department of Geomatics \\ Engineering Schulich School of Engineering, University of Calgary, Calgary, Canada) \\ (E-mail: xiefriday@gmail.com)
}

\begin{abstract}
Multipath arises from the reception of reflected or diffracted signals in addition to the LineOf-Sight (LOS) signal. By using a block processing high sensitivity receiver scheme, this paper aims to obtain better positioning performance in urban canyon areas. Generally, the peak with the most power is utilised in high sensitivity receivers; however, this approach is not always optimal in multipath environments. Noting that signal correlation peaks may be separated in the Doppler domain by a long coherent integration time, a peak identification scheme is proposed in this work, which yields better positioning performance. It is shown that most of the multipath peaks are removed in the receiver after using the proposed algorithm.
\end{abstract}

\section{KEYWORDS}
1. High Sensitivity.
2. Coherent Integration.
3. Urban Canyon.
4. Multipath Signal

Submitted: 26 October 2014. Accepted: 23 February 2015. First published online: 26 March 2015.

1. INTRODUCTION. Generally, standard Global Navigation Satellite System (GNSS) receiver architectures cannot provide an accuracy level sufficient for use in vehicular applications in all environments. This is especially true for urban canyon environments, where the presence of large buildings leads to frequent shadowing/ attenuation of signals as well as the reception of several Non-Line-Of-Sight (NLOS) signals. The latter can result in several identifiable peaks in the receiver's correlator outputs (Soloviev and van Graas, 2008).

Because of the signal degradation and/or multiple received signal paths in these adverse environments, high sensitivity receivers have been developed. Such receivers commonly employ block processing techniques which have been presented in various publications (Psiaki, 2001; Gunawardena et al., 2004; Van Graas et al., 2005; Soloviev and van Graas., 2008; O’Driscoll et al., 2011). Block processing uses a larger grid of correlators; the signal parameters (i.e., code phase and carrier Doppler) are estimated directly from the correlator outputs.

In the block processing strategy, generally the assumption is that the Line-Of-Sight (LOS) signal is stronger than the multipath signals. To this end, usually the correlator with the most power (i.e., dominant peak) is selected as the LOS signal (O'Driscoll et al., 2011; Lin et al., 2011). However, in some scenarios it is not assured that the 
dominant peak is the LOS peak. For example, in urban canyons, it is possible that the LOS signal is highly attenuated by buildings while a strong reflected signal is also received. In this case, the initial assumption is invalid and the dominant peak is not the LOS peak. In other words, different means of identifying LOS signals need to be developed to mitigate the effect of signal reflections (multipath) and the corresponding position errors.

Previous research shows that, in general, the LOS signal and NLOS signals have different Doppler frequencies (Soloviev and van Graas, 2008; O’Driscoll et al., 2011). Further to this, the correlation peak corresponding to LOS and NLOS signals can be separated in the frequency domain by a long coherent integration time (Soloviev and van Graas, 2008; O'Driscoll et al., 2011; Xie et al., 2011; Xie and Petovello, 2014). Finally, Xie and Petovello (2014) showed that Doppler shifts of NLOS signals are dependent on the direction of motion of the user. Collectively, these findings motivate the separation of the LOS and NLOS signals in a vector-based, block processing receiver with specific application to vehicular navigation in urban environments.

Two methods are proposed in this work to better identify LOS signals. In particular, the main contribution of this paper is a method to identify the "best" peak - assumed to be the LOS peak - in the correlator block in order to reduce errors from NLOS signals. This is done by using the receiver's Position, Velocity and Time (PVT) estimates to define regions where LOS and NLOS signals are likely to reside. These regions provide an additional means (beside correlator power) to identify LOS or NLOS signals. At the same time, because the receiver uses vector-tracking concepts (Parkinson and Spilker, 1996; Lashley and Bevly, 2008), the peak identification algorithm is susceptible to position and velocity errors. This paper extends the work in Xie and Petovello (2014) to accommodate the uncertainty in the receiver's PVT estimates. These regions are then used to identify the "best" correlator peak. The algorithm is tested in high multipath environments and is shown to provide considerable position and velocity improvements over traditional high-sensitivity tracking approaches.

The paper begins with a brief review of the relevant background and methodologies. Next, two strategies for identifying the LOS signal are proposed and LOS region and NLOS region are derived. Also, a strategy to determine possible errors in the navigation solution is proposed for the code phase and Doppler domains. The paper then describes the data collections and analyses the results using different receiver architectures. Relevant conclusions are then drawn based on the results.

2. BACKGROUND. This section briefly presents the relevant background information used in the development of the proposed algorithms described in the next section.

2.1. NLOS Doppler region. To begin, the Doppler frequency of a received GNSS signal can be expressed as (Misra and Enge, 2006; Kaplan and Hegarty, 2006):

$$
f_{D}=\frac{\vec{v}_{S} \cdot \vec{h}_{L O S}}{\lambda}-\frac{\vec{v}_{R} \cdot \vec{h}_{R}}{\lambda}+\frac{d_{R}}{\lambda}
$$

where $\vec{v}_{S}$ is the satellite velocity vector; $\vec{v}_{R}$ is the receiver velocity vector; $\vec{h}_{L O S}$ is the LOS unit vector pointing from the receiver to the satellite; $\vec{h}_{R}$ is the unit vector pointing in the direction of the received signal ("signal vector"); $d_{R}$ is the receiver clock drift in $\mathrm{m} / \mathrm{s}$; and $\lambda$ is the signal wavelength. It is noted that the first term is the contribution 
from satellite motion whereas the second term is the contribution from receiver motion.

For the LOS case, the signal vector is equal to the LOS vector such that

$$
\left.\vec{h}_{R}\right|_{L O S}=\vec{h}_{L O S}
$$

For the NLOS case, the signal vector points in the direction of the reflector such that

$$
\left.\vec{h}_{R}\right|_{N L O S}=\vec{h}_{N L O S}
$$

where $\vec{h}_{N L O S}$ points towards the reflection point of the signal.

Using Equation (1) as a starting point, Xie and Petovello (2014) showed that reflected signals have Doppler values that fall within the following NLOS region

$$
M P \in\left[\frac{1}{\lambda}\left(\vec{v}_{R} \cdot \vec{h}_{L O S}-\left|\vec{v}_{R}\right|\right), \frac{1}{\lambda}\left(\vec{v}_{R} \cdot \vec{h}_{L O S}+\left|\vec{v}_{R}\right|\right)\right]
$$

The key point of the above equation is that, because of the dot product involving the receiver velocity, the minimum and maximum Doppler value in the multipath region is not, in general, of equal magnitude. It also follows that the NLOS region depends on the direction of travel of the user.

2.2. NLOS distributions in an urban canyon environment. In the context of this paper, the following aspects of reflected signals (with particular emphasis on urban environments) are of importance:

1. The code phase and Doppler accuracies are highly correlated with the signal to noise density ratio $\left(\mathrm{C} / \mathrm{N}_{0}\right)$, with higher $\mathrm{C} / \mathrm{N}_{0}$ signals yielding more accurate measurements (Langley, 1997, Xie and Petovello, 2014).

2. The effect of multipath on Doppler measurements is related to the receiver's velocity vector (see Equation (4)). Also, longer coherent integration time has better peak separation performance (Xie and Petovello, 2014).

3. NLOS peaks are direction-dependent, so given the satellite geometry and vehicle velocity, the multipath region can be predicted. The equations to predict multipath regions are given in Equation (4).

4. The correlator peak with the largest magnitude does not always correspond to the LOS signal. In some cases, the power of reflected signals can be stronger than the LOS signal, but is usually lower than $42 \mathrm{~dB}-\mathrm{Hz}$ (Xie and Petovello, 2014).

2.3. Vector-based block processing strategy. The vector-based strategy employed in this research is shown in Figure 1. At each epoch, the estimated position and velocity from the navigation filter is used to generate the nominal code phase, $\tau_{0}$, and Doppler, $f_{0}$, of the LOS signal (Lashley and Bevly, 2008), which are used to set the code phase and frequency of the receiver's Numerically Controlled Oscillators (NCOs). Then, the receiver generates a correlator grid, or search space, wherein the locally generated signals span $\hat{\tau} \in\left[\tau_{0}-S_{\tau}, \quad \tau_{0}+S_{\tau}\right]$ in the code phase domain and $\hat{f}_{D} \in$ $\left[f_{0}-S_{f}, f_{0}+S_{f}\right]$ in the frequency domain, where a "hat" indicates a locally generated value, $S_{\tau}$ and $S_{f}$ define the search range, or search space size, in the code phase and frequency domain respectively. 


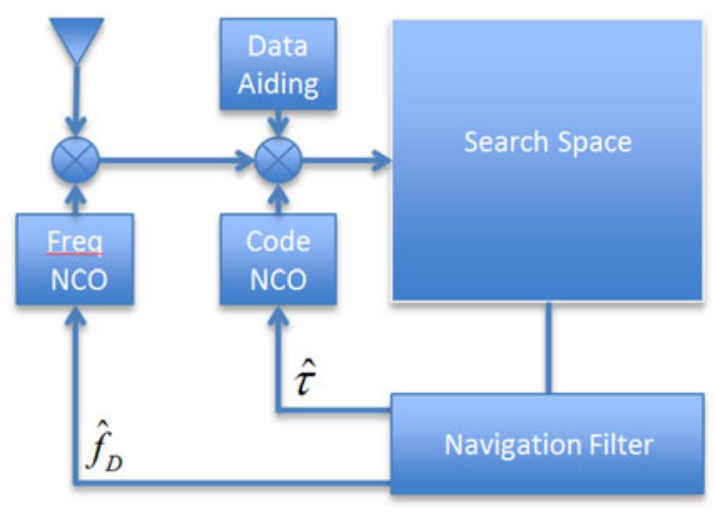

Figure 1. Illustration of vector-based strategy employed in this work.

It is noted that the receiver's PVT estimates are used to define the size of the search space. It follows therefore that any uncertainties in the PVT estimates should be accounted for. At the same time, large errors in the PVT estimates will impact the exact location within the search space where the received signal is expected to reside, and thus should also be considered. These concepts are discussed in the proposed peak identification strategies section.

2.4. Dominant peak strategy. The commonly used approach of selecting the dominant peak in the block processing method (O'Driscoll et al., 2011) is herein called the dominant peak strategy. For this strategy, the receiver only needs to identify the correlator with the maximum power - the dominant peak-from the correlation map. However, the drawback of this strategy is that a NLOS signal can be easily considered as a LOS signal. Xie et al. (2011) stated that in the urban canyon area the dominant peak is not the LOS peak more than 20 per cent of the time. By extension, unless additional information is used, dominant NLOS peaks will be considered as LOS peaks and large positioning errors will be introduced in the receiver. In this regard, two improved LOS peak identification strategies are proposed in the next section to better identify the LOS signals.

3. PROPOSED PEAK IDENTIFICATION STRATEGIES. The first step in the proposed method is to identify the various signal peaks in the search space. Different thresholds can be used for this purpose (Misra and Enge, 2006; Borio et al., 2008). In this work, any correlator peak that corresponds to a signal with a $\mathrm{C} / \mathrm{N}_{0}$ of $16 \mathrm{~dB}-\mathrm{Hz}$ or less (accounting for the coherent integration time) is assumed to be due to noise and is called a noise peak (Xie, 2013). Any other peak is considered a signal peak, and the challenge is to select signal peaks that correspond to LOS paths only.

With this in mind, two different approaches are proposed. The first strategy tries to distinguish LOS and NLOS signals based on the received signal power as determined by the magnitude of the correlator's output. Correspondingly, it is referred to as the power-based strategy. The second method expands on the first by also considering regions within the search space where LOS signals would be expected to be located 
Table 1. Region-based LOS Peak Identification Strategy.

\begin{tabular}{lcll}
\hline Classification & $\mathrm{C} / \mathrm{N}_{0}(\mathrm{~dB}-\mathrm{Hz})$ & Doppler Shift $(\mathrm{Hz})$ & Code Shift \\
\hline LOS & $\geq 42$ & Nhips $)$ \\
& $\geq 16$ & $\in \mathcal{L}_{f}$ & N/A \\
Multipath & $\geq 16$ & $\notin \mathcal{L}_{f, L O S}$ & $\in \mathcal{L}_{\tau, L O S}$ \\
Cross-Correlation & $\geq 16$ & N/A & $\notin \mathcal{L}_{\tau, L O S}$ \\
\hline
\end{tabular}

and uses these regions to further improve LOS identification. The second strategy is referred to as the region-based strategy.

The two strategies are discussed in more detail in the following sub-sections.

3.1. Power-based strategy. It was reported in previous work that multipath signal power can be stronger than the LOS signal, but most of the multipath signal powers are lower than $42 \mathrm{~dB}-\mathrm{Hz}$ (Xie and Petovello, 2014). The power-based strategy thus assumes that if a signal peak power is more than $42 \mathrm{~dB}-\mathrm{Hz}$, then it corresponds to the LOS signal. In other words, code phase and Doppler measurements are only generated from those peaks whose signal powers are stronger than $42 \mathrm{~dB}-\mathrm{Hz}$.

The benefit of this approach is its simplicity; it can be implemented in existing receivers using a firmware update. The major drawback of this strategy, however, is that the receiver may not always identify enough LOS signals to compute a solution, especially in urban canyons.

3.2. Region-based strategy. The second strategy expands on the power-based strategy by defining a region within the search space called the LOS region where the LOS signals should, under ideal conditions, be located, if present. The strategy is summarised in Table 1, where $\mathcal{L}_{f}$ represents the LOS region in the Doppler domain, and $\mathcal{L}_{\tau}$ represents the LOS region in the code phase domain. These regions will be derived below.

In light of Table 1, a possible LOS peak is declared present when a peak has a signal power larger than $42 \mathrm{~dB}-\mathrm{Hz}$ (similar to power-based strategy), or when the peak falls within the LOS region in the code phase and Doppler domains. A multipath peak is declared when a peak falls outside of the LOS region either in the code phase or Doppler domain. It is noted that the cross-correlation peaks should be removed from the correlation map, which was discussed in Xie and Petovello (2012). The following sub-sections derive the relevant equations for the LOS and NLOS regions; this is an extension of Xie (2013) and represents one of the main contributions of the work.

3.2.1. LOS and NLOS regions in Doppler domain. Estimating the Doppler frequency requires the receiver's PVT estimates from its navigation filter. Position errors have negligible effect on the computed Doppler and are thus ignored. The uncertainty in the estimated velocity and clock drift, however, must be treated explicitly. The estimated Doppler, $\hat{f}_{D}$, is obtained from Equation (1) by replacing the true receiver velocity and clock drift with their respective estimated values, $\hat{\vec{v}}_{R}$ and $\hat{d}_{R}$, as follows (Xie, 2013):

$$
\hat{f}_{D}=\frac{\vec{v}_{S} \cdot \vec{h}_{L O S}}{\lambda}-\frac{\hat{\vec{v}}_{R} \cdot \vec{h}_{R}}{\lambda}+\frac{\hat{d}_{R}}{\lambda}
$$

It is noted that satellite velocity is considered perfect since it can be calculated precisely by using the broadcast ephemeris (Zhang et al., 2006). The difference, or offset, of the 
estimated and true LOS Doppler can be computed as:

$$
\begin{aligned}
\Delta f_{D} & =\hat{f}_{D}-\left.f_{D}\right|_{\vec{h}_{R}=\vec{h}_{L O S}} \\
& =\frac{1}{\lambda}\left(\vec{v}_{R} \cdot \vec{h}_{L O S}-\hat{\vec{v}}_{R} \cdot \vec{h}_{R}+\delta \hat{d}_{R}\right)
\end{aligned}
$$

where $\delta \hat{d}_{R}=\hat{d}_{R}-d_{R}$, is the error in the estimated clock drift. It is noted that the first term in Equation (6) is the true Doppler (due to user motion), and thus does not depend on the estimated velocity. Recalling Equations (2) and (3), the Doppler offsets for the LOS and NLOS scenarios are given respectively as

$$
\begin{gathered}
\Delta f_{D, L O S}=\frac{1}{\lambda}\left(\vec{v}_{R} \cdot \vec{h}_{L O S}-\hat{\vec{v}}_{R} \cdot \vec{h}_{L O S}+\delta \hat{d}_{R}\right) \\
\Delta f_{D, N L O S}=\frac{1}{\lambda}\left(\vec{v}_{R} \cdot \vec{h}_{L O S}-\hat{\vec{v}}_{R} \cdot \vec{h}_{N L O S}+\delta \hat{d}_{R}\right)
\end{gathered}
$$

These equations hold in general, but Equation (8) is of little practical use because the NLOS signal vector is generally unknown (i.e., $\vec{h}_{N L O S}$ ). Instead, by realizing that $\left|\hat{\vec{v}}_{R}\right| \geq \hat{\vec{v}}_{R} \cdot \vec{h}_{N L O S}$, the NLOS region for signal Doppler, $\mathcal{N}_{f}$, is given by

$$
\mathcal{N}_{f} \in\left[\begin{array}{c}
\frac{1}{\lambda}\left(\vec{v}_{R} \cdot \vec{h}_{L O S}-\left|\hat{\vec{v}}_{R}\right|+\delta \hat{d}_{R}\right), \\
\frac{1}{\lambda}\left(\vec{v}_{R} \cdot \vec{h}_{L O S}+\left|\hat{\vec{v}}_{R}\right|+\delta \hat{d}_{R}\right)
\end{array}\right]
$$

Note that the upper bound and lower bounds are generally not the same in terms of the absolute value; in other words, multipath is directionally-dependent (Xie and Petovello, 2012; Xie, 2013).

Equations (7) and (9) represent the first-order approximation of the LOS and NLOS regions, respectively (note that in the absence of errors, the LOS region degenerates to a single value). From a practical perspective, what remains is to consider the ability of a receiver to compute these regions on the fly. We begin by assuming that the velocity and clock drift estimates are unbiased such that $E\left(\hat{\vec{v}}_{R}\right)=\vec{v}_{R}$ and $E\left(\hat{d}_{R}\right)=d_{R}$. The case where this is not true is considered later in the paper.

The expectation of the Equations (7) and (9) can be respectively given as

$$
E\left(\Delta f_{D, L O S}\right)=0
$$

and

$$
E\left(\mathcal{N}_{f}\right) \in\left[\begin{array}{c}
\frac{1}{\lambda}\left(\vec{v}_{R} \cdot \vec{h}_{L O S}-\left|\hat{\vec{v}}_{R}\right|\right) \\
\frac{1}{\lambda}\left(\vec{v}_{R} \cdot \vec{h}_{L O S}+\left|\hat{\vec{v}}_{R}\right|\right)
\end{array}\right]
$$

As $\vec{v}_{R}$ is generally unknown, the best approximation is $\vec{v}_{R} \approx \hat{\vec{v}}_{R}$, and thus Equation (11) 
can be rewritten as

$$
E\left(\mathcal{N}_{f}\right) \in\left[\begin{array}{c}
\frac{1}{\lambda}\left(\hat{\vec{v}}_{R} \cdot \vec{h}_{L O S}-\left|\hat{\vec{v}}_{R}\right|\right), \\
\frac{1}{\lambda}\left(\hat{\vec{v}}_{R} \cdot \vec{h}_{L O S}+\left|\hat{\vec{v}}_{R}\right|\right)
\end{array}\right]
$$

Next, consider the uncertainty of the velocity and clock drift parameters. Their covariance matrix, denoted as $P_{v d}$, can be extracted from the navigation filter and propagated into the Doppler domain as follows

$$
\sigma_{\Delta f}^{2}=\frac{\left[\begin{array}{ll}
\vec{h}_{L O S} & 1
\end{array}\right] P_{v d}\left[\begin{array}{ll}
\vec{h}_{L O S} & 1
\end{array}\right]^{T}}{\lambda^{2}}
$$

where $P_{v d}=\operatorname{var}\left(\left[\begin{array}{ll}\hat{\vec{v}}_{R} & \hat{d}_{R}\end{array}\right]\right)$.

Furthermore, given the vehicle velocity and satellite geometry (i.e., $\hat{\vec{h}}_{L O S}$ ), the LOS and NLOS regions should be expanded as follows

$$
\begin{aligned}
\mathcal{L}_{f}=\left[\begin{array}{ll}
-m \sigma_{\Delta f}, & m \sigma_{\Delta f}
\end{array}\right] \\
\left(\mathcal{N}_{f}\right) \in\left[\begin{array}{l}
\frac{1}{\lambda}\left(\hat{\vec{v}}_{R} \cdot \hat{\vec{h}}_{L O S}-\left|\hat{\vec{v}}_{R}\right|\right)-m \sigma_{\Delta f}, \\
\frac{1}{\lambda}\left(\hat{\vec{v}}_{R} \cdot \hat{\vec{h}}_{L O S}+\left|\hat{\vec{v}}_{R}\right|\right)+m \sigma_{\Delta f}
\end{array}\right]
\end{aligned}
$$

where $m$ is a scale factor which indicates the confidence level, e.g., $m=1$ for $68 \cdot 3 \%$ and $m=2$ for $95.4 \%$.

3.2.2. LOS region in code phase domain. Similar to the Doppler domain analysis, the LOS region in the code phase domain when accounting for uncertainty in the receiver's PVT estimates is given by (Xie, 2013)

$$
\mathcal{L}_{\tau}=\left[-m \sigma_{\Delta_{\tau}}, \quad m \sigma_{\Delta_{\tau}}\right]
$$

The variance is given by

$$
\sigma_{\Delta \tau}^{2}=\frac{\left[\begin{array}{ll}
\vec{h}_{L O S} & 1
\end{array}\right] P_{p b}\left[\begin{array}{ll}
\vec{h}_{L O S} & 1
\end{array}\right]^{T}}{L^{2}}
$$

where $P_{p b}=\operatorname{var}\left(\left[\begin{array}{ll}\hat{\vec{P}}_{R} & \hat{b}_{R}\end{array}\right]\right)$ can be obtained from the navigation Kalman filter directly. $\vec{P}_{R}$ is the receiver position vector; $b_{R}$ is the receiver clock bias in metres; and $L$ is code chip length.

Notice that an NLOS region in the code phase domain can be defined but is of little practical use. The reason is because, theoretically, the NLOS code phase delay could be infinite. As such, only the LOS region is employed for the code phase domain in this work.

3.2.3. Receiver anomaly detection scheme. A vital challenge for the region-based strategy is to be tolerant to errors in the receiver's PVT estimates. Whereas the LOS and NLOS regions defined in the previous section account for uncertainty (based on a standard deviation), this section focuses on the presence of large errors that cannot be adequately accounted for using a single standard deviation value and that cause a shift in the correlator's grids (and corresponding LOS and NLOS regions). 
Here, large errors in the receiver's PVT estimates are collectively referred to as receiver anomalies. When present, the correlator grids will be shifted in the Doppler and/or code phase domains. Furthermore, if the shift is sufficiently large, one or more of the following cases may result:

- The LOS signal may not fall within the LOS region (missed-detection of LOS signal)

- NLOS signals may not fall within the NLOS region

- NLOS signals may fall within the LOS region (false-detection of LOS signal).

With this in mind, a receiver anomaly detection scheme is proposed based on the location and power of peaks observed in the correlator grid. If a signal with a $\mathrm{C} / \mathrm{N}_{0}$ of $42 \mathrm{~dB}-\mathrm{Hz}$ or larger is detected (and thus assumed to be LOS) and is located outside the LOS region, either in the code phase domain or in the Doppler domain, a receiver anomaly (or receiver bias) in the position or velocity is declared. Moreover, if a NLOS signal is identified to be outside the Doppler region in the Doppler domain, a receiver anomaly in the velocity domain is declared.

However, as biases in the nominal signal parameters are generally unknown, the LOS and NLOS regions are scaled until all peaks fall within the refined regions. For example, if the observed NLOS peak is outside the predicted NLOS region, the scale factor $m$ is increased so that the predicted region contains all the peaks. It is noted that the scale factor $m$ is reset to the original value (three in this work) at every epoch after LOS peak identification. This is consistent with the idea that the anomalies being detected occur relatively infrequently.

The receiver anomaly detection process is broken down into two parts; one for position anomalies and another for velocity anomalies. The position anomaly detection scheme is summarised in Figure 2. LOS peak code phase offset can be utilised to diagnose the position anomaly in the receiver. If the LOS peak falls in the predicted code phase region, no position anomaly is reported. Alternatively, if the LOS peak falls outside of the predicted region in Equation (16), a position anomaly will be reported and, correspondingly, the LOS region in the code phase domain should be refined. Note that the position anomaly is difficult to identify as only the LOS signal can be used.

In general, velocity anomalies are easier to detect than position anomalies due to the fact that both LOS and multipath peaks can be used. If the identified peak is the LOS peak (i.e., $\mathrm{C} / \mathrm{N}_{0}$ is larger than $42 \mathrm{~dB}-\mathrm{Hz}$ ), the Doppler offset associated with this peak is compared to the predicted LOS region. In contrast, if the peak is a NLOS peak, the Doppler offset associated with this peak is compared to the predicted NLOS region. Finally, if any peak is outside of its predicted region, a velocity anomaly is reported and the peak regions will be refined. The receiver velocity anomaly detection scheme is summarised in Figure 3.

4. RESULTS AND ANALYSIS. To assess the proposed algorithms in vehicular applications, live data was collected in downtown Calgary, Canada. Data collection started with a static open-sky period of approximately three minutes that was used to initialise the receiver's position and velocity. A NovAtel SPAN system with a tactical grade UIMU-LCI inertial sensor ("SPAN LCI") was used to generate the reference trajectory using NovAtel Inertial Explorer software. A National Instrument (NI) 


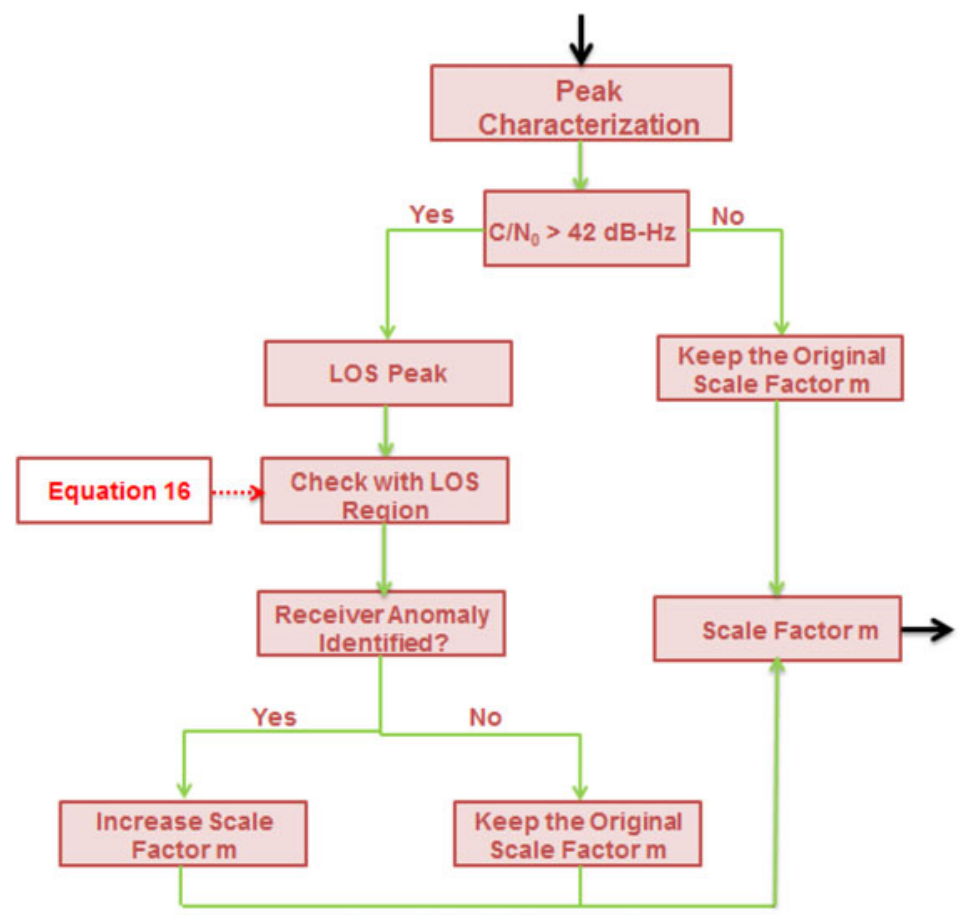

Figure 2. Proposed receiver position anomaly check strategy in the high sensitivity receiver.

RF front-end was used to collect the IF data from the same antenna. For the GPS L1 signal the NI local oscillator frequency was $1575 \mathrm{MHz}$ (intermediate frequency of $0.42 \mathrm{MHz}$ ), the bandwidth was $10 \mathrm{MHz}$, and the sampling rate was $12.5 \mathrm{Msps}$ (complex).

The estimated accuracy ( $1 \sigma$, from NovAtel Inertial Explorer outputs) of the SPAN LCI solution during the data collection were less than $0.5 \mathrm{~m}$, and the velocity errors are less than $1 \mathrm{~cm} / \mathrm{s}$. Moreover, the oscillator in the NI front-end is a very stable Oven Controlled Crystal (OCXO) with a short-term frequency stability better than 1e-11 (Gaggero, 2008), thus the frequency uncertainty introduced by the oscillator can be ignored (Gaggero and Borio, 2008). In this regard, the reference trajectory from SPAN LCI can be utilised to generate the nominal LOS signal parameters, which can be used to verify if the identified LOS signal corresponds to the LOS signal. This is used for assessing measurement errors later in the paper.

Availability and reliability are defined in this work to evaluate the peak identification performance. Availability refers to the percentage of time an observation of a satellite can be generated, be it from a LOS or multipath signal. Reliability is the percentage of time an available measurement corresponds to a LOS signal. In other words, a satellite may have $50 \%$ availability, but if all measurements are from LOS signals, the reliability would be $100 \%$.

The block processing parameters applied in this work are shown in Table 2. The search space size applied is $\pm 30 \mathrm{~Hz}$ in the Doppler domain, and $\pm 150 \mathrm{~m}$ in the code phase domain, respectively. The coherent integration time was $200 \mathrm{~ms}$ (using bit 


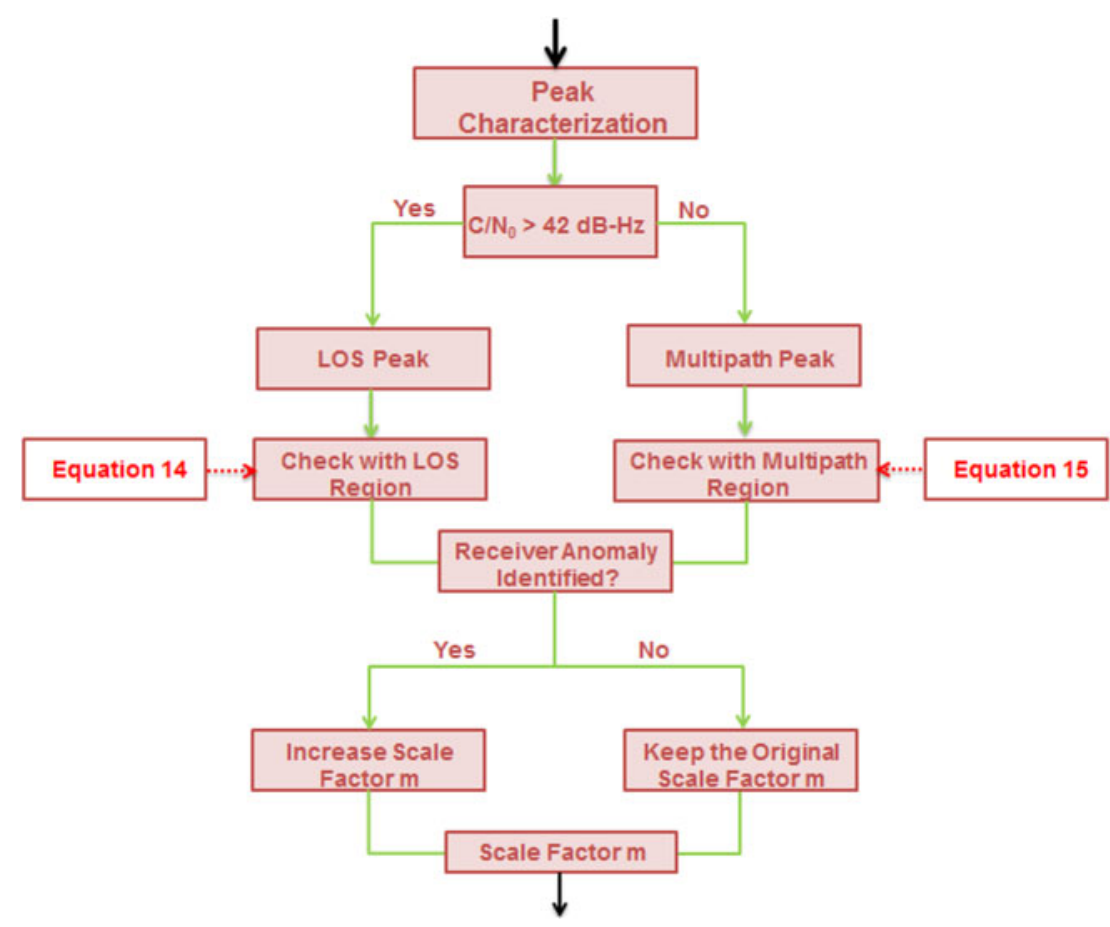

Figure 3. Proposed receiver velocity anomaly check strategy in the high sensitivity receiver.

Table 2. Block processing parameters.

\begin{tabular}{lll}
\hline Integration Time & $200 \mathrm{~ms}$ & \\
\hline Search Space & Doppler Domain & $\pm 30 \mathrm{~Hz}$ \\
& Code Phase Domain & $\pm 150 \mathrm{~m}$ \\
Search Step & Doppler Domain & $2 \mathrm{~Hz}$ \\
& Code Phase Domain & $10 \mathrm{~m}$ \\
\hline
\end{tabular}

aiding from a nearby receiver) and the frequency resolution is $5 \mathrm{~Hz}$ (Xie and Petovello, 2014). The search step in the code phase domain and Doppler domain is $10 \mathrm{~m}$ and 2 $\mathrm{Hz}$, respectively.

4.1. Dominant peak strategy. A high-sensitivity receiver using the dominant peak strategy is evaluated in this section. Specifically, longer coherent integration time compared to the standard receiver is applied (i.e., longer than $20 \mathrm{~ms}$ ). Figure 4 shows the position and velocity performance for a $200 \mathrm{~ms}$ coherent integration time. A relatively large position error variation is observed during the period from $423100 \mathrm{~s}$ to $423300 \mathrm{~s}$, and again, during the period from $423550 \mathrm{~s}$ to $423600 \mathrm{~s}$. This is due to multipath signals with large pseudorange errors being considered as the LOS peaks in the receiver. In addition, the velocity errors are less than $1 \mathrm{~m} / \mathrm{s}$ most of the time and reach a maximum of $6 \mathrm{~m} / \mathrm{s}$. In such cases, since vector-tracking is used, the receiver NCO frequency can be shifted by a few $\mathrm{Hz}$ to tens of $\mathrm{Hz}$ relative to the actual value. 

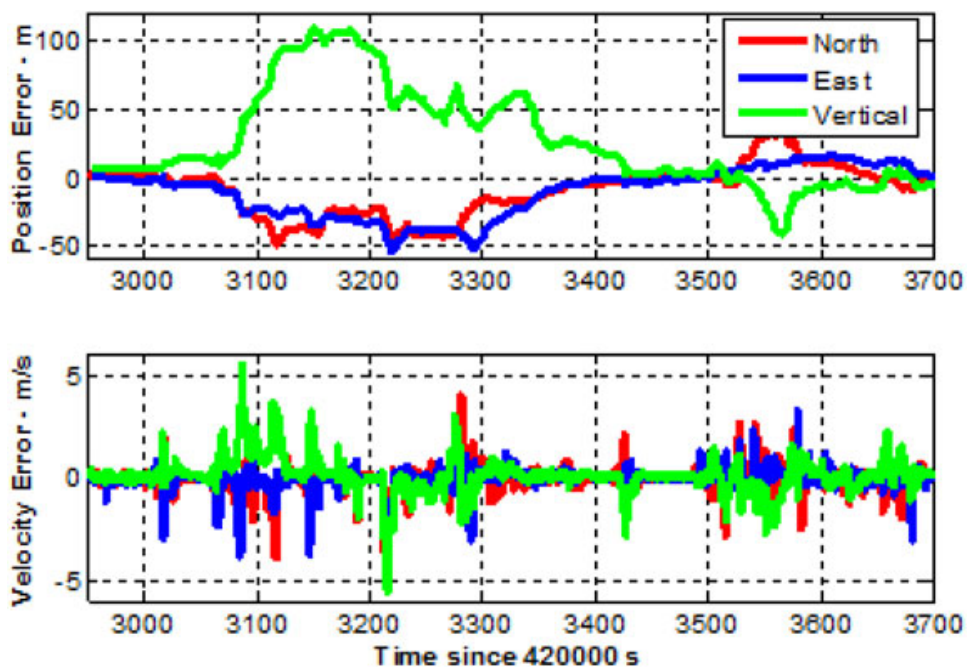

Figure 4. Position and velocity performance for $200 \mathrm{~ms}$ coherent integration of the dominant peak strategy using Kalman filter.
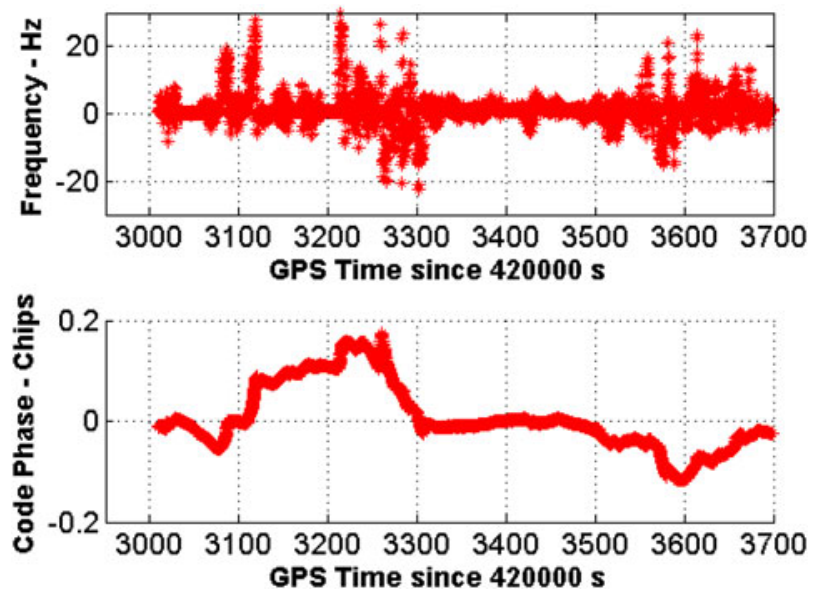

Figure 5. PRN 4 NCO frequency and code phase errors by using the dominant peak strategy.

PRN 4 is selected here to show the NCO errors during the performed test, but results are indicative of other satellites. In this context, the NCO errors represent the Doppler offset and code phase offset of the LOS peak, if present. Figure 5 shows the NCO code phase and frequency errors of PRN 4, whose elevation and azimuth are $50^{\circ}$ and $153^{\circ}$, respectively. It is observed that the NCO frequency errors (in terms of the absolute value) are less than $10 \mathrm{~Hz}$ most of the time, and jump to 20 to $30 \mathrm{~Hz}$ in extreme cases. Also, the NCO code phase errors are generally less than $0 \cdot 1$ chips and jump to $0 \cdot 15$ chips for some epochs. 
Table 3. Measurement Quality from the Dominant Peak Strategy.

\begin{tabular}{lcccccccc}
\hline & & \multicolumn{2}{l}{ Pseudorange Error } & & \multicolumn{2}{c}{ Doppler Error } & & \\
PRN & Elevation $(\mathrm{deg})$ & Mean $(\mathrm{m})$ & STD $(\mathrm{m})$ & & Mean $(\mathrm{Hz})$ & STD $(\mathrm{Hz})$ & Availability & Reliability \\
\hline 2 & 22 & $-6 \cdot 0$ & $30 \cdot 6$ & & $-0 \cdot 4$ & $3 \cdot 4$ & $77 \%$ & $83 \%$ \\
4 & 50 & $-0 \cdot 8$ & $15 \cdot 4$ & & $0 \cdot 2$ & $2 \cdot 6$ & $92 \%$ & $80 \%$ \\
9 & 54 & $-0 \cdot 5$ & $18 \cdot 6$ & & $-0 \cdot 4$ & $3 \cdot 3$ & $83 \%$ & $78 \%$ \\
12 & 30 & $-26 \cdot 7$ & $45 \cdot 3$ & & $0 \cdot 2$ & $5 \cdot 9$ & $59 \%$ & $54 \%$ \\
17 & 53 & $-0 \cdot 0$ & $21 \cdot 3$ & & $0 \cdot 5$ & $4 \cdot 8$ & $91 \%$ & $78 \%$ \\
27 & 53 & $-3 \cdot 6$ & $20 \cdot 6$ & & $-1 \cdot 0$ & $4 \cdot 6$ & $73 \%$ & $70 \%$ \\
28 & 24 & $-23 \cdot 4$ & $45 \cdot 0$ & & $-0 \cdot 3$ & $4 \cdot 9$ & $61 \%$ & $62 \%$ \\
\hline
\end{tabular}
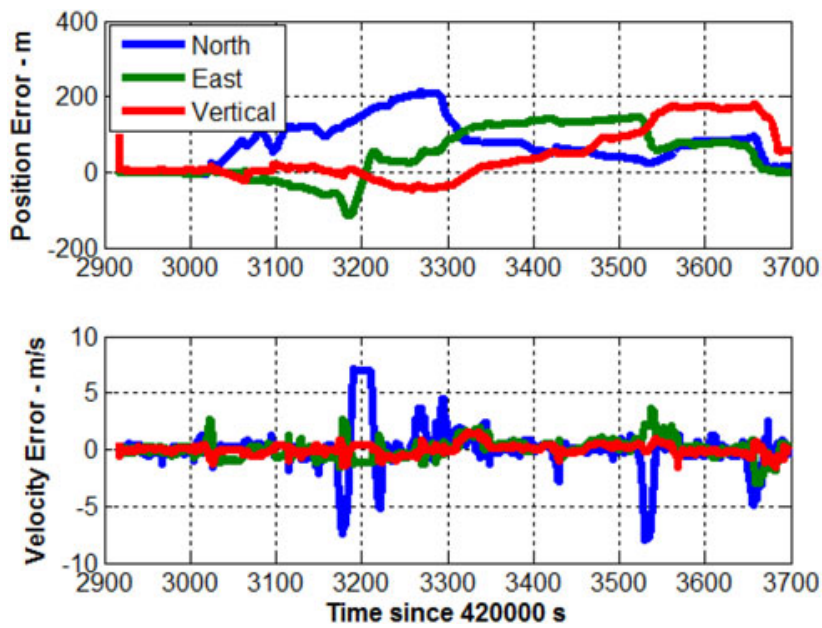

Figure 6. Position and velocity performance of the power-based strategy for $200 \mathrm{~ms}$ coherent integration by employing Kalman filter.

Table 3 shows the availability and reliability of measurements when using the dominant peak method. Low elevation satellites generally have poor measurement qualities (e.g., PRN 12 and PRN 28) with relatively large pseudorange errors and Doppler errors. The availability for PRN 12 is $59 \%$, which means that only $59 \%$ of the time the signal (either LOS signal or multipath signal) is present in the correlation map. Only $54 \%$ of these signal peaks are actually the LOS signals. In contrast, for the high elevation satellites (e.g., PRN 4 and PRN 17), the availability is more than $80 \%$, and around $80 \%$ of them are the LOS signals.

4.2. Power-based strategy. Recall that the idea behind the power-based strategy is to only use strong signals in the urban canyon environment, i.e., stronger than $42 \mathrm{~dB}$ Hz. Figure 6 shows the position and velocity performance for a $200 \mathrm{~ms}$ coherent integration time (the same processing parameters shown in Table 2 are used.). Degraded position and velocity performance are observed in this figure compared to the dominant peak strategy. This is due to the receiver not being able to identify enough LOS signals in the weak signal scenarios. In fact, this is the major drawback of this approach. 

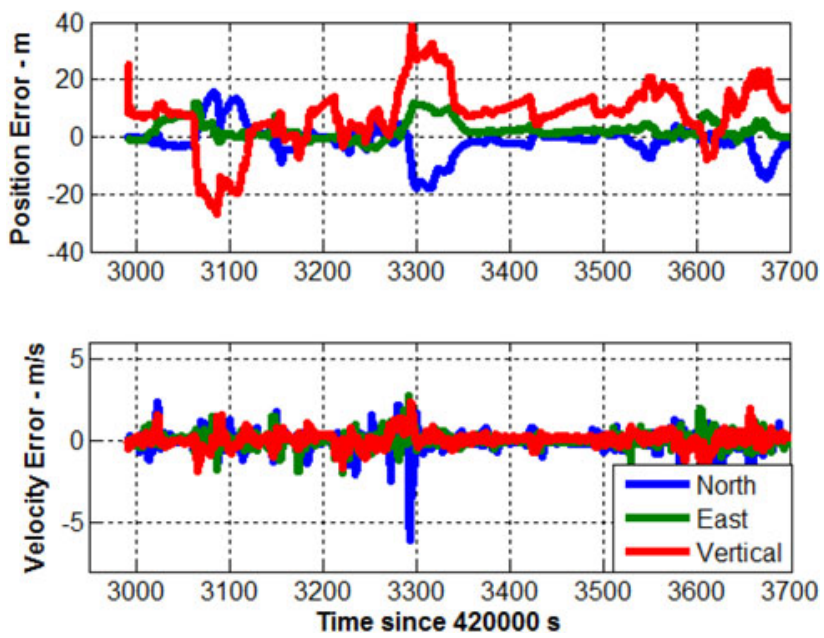

Figure 7. Position and velocity performance of the region-based strategy for $200 \mathrm{~ms}$ coherent integration by employing Kalman filter.

Table 4. RMS North, East and vertical (N, E, V) position and velocity errors using different receiver strategies.

\begin{tabular}{|c|c|c|c|c|c|c|}
\hline \multirow[b]{2}{*}{ Receiver Type } & \multicolumn{3}{|c|}{ Position (m) } & \multicolumn{3}{|c|}{ Velocity (m/s) } \\
\hline & $\mathrm{N}$ & $\mathrm{E}$ & $\mathrm{V}$ & $\mathrm{N}$ & $\mathrm{E}$ & $\mathrm{V}$ \\
\hline Commercial & $34 \cdot 9$ & $43 \cdot 4$ & $32 \cdot 3$ & $3 \cdot 32$ & $2 \cdot 92$ & $0 \cdot 65$ \\
\hline Dominant Peak & $26 \cdot 7$ & $27 \cdot 7$ & $61 \cdot 2$ & $1 \cdot 58$ & $1 \cdot 17$ & $1 \cdot 36$ \\
\hline Region-based & $4 \cdot 1$ & $5 \cdot 6$ & $18 \cdot 5$ & $0 \cdot 44$ & $0 \cdot 37$ & $0 \cdot 46$ \\
\hline
\end{tabular}

4.3. Region-based strategy. The position performance of the region-based strategy is shown in Figure 7. As expected, this strategy has better performance than the dominant peak strategy and power-based strategy (shown in Figures 4 and 6, respectively). Whereas the horizontal position errors were bounded by about $50 \mathrm{~m}$ for the previous two strategies, the region-based strategy limits the horizontal errors to less than $20 \mathrm{~m}$.

Large velocity errors are also observed in Figure 7 for several epochs. These result from false identification of NLOS peaks as LOS peaks. Notwithstanding these few cases, overall, the errors are much smaller than for the dominant or power-based strategies.

Table 4 summarises the position and velocity error statistics. The performance of the commercial high sensitivity receiver and the dominant peak strategy are also shown for comparison. It is observed that the position accuracy is improved substantially after using the region-based strategy.

4.3.1. Receiver anomaly detection performance. This section demonstrates the change in the LOS region before and after applying the anomaly detection algorithm. By extension, it demonstrates the ability of the algorithm to mitigate the effect of NLOS signals on the final solution. PRN 4 is selected as an illustrative example. 


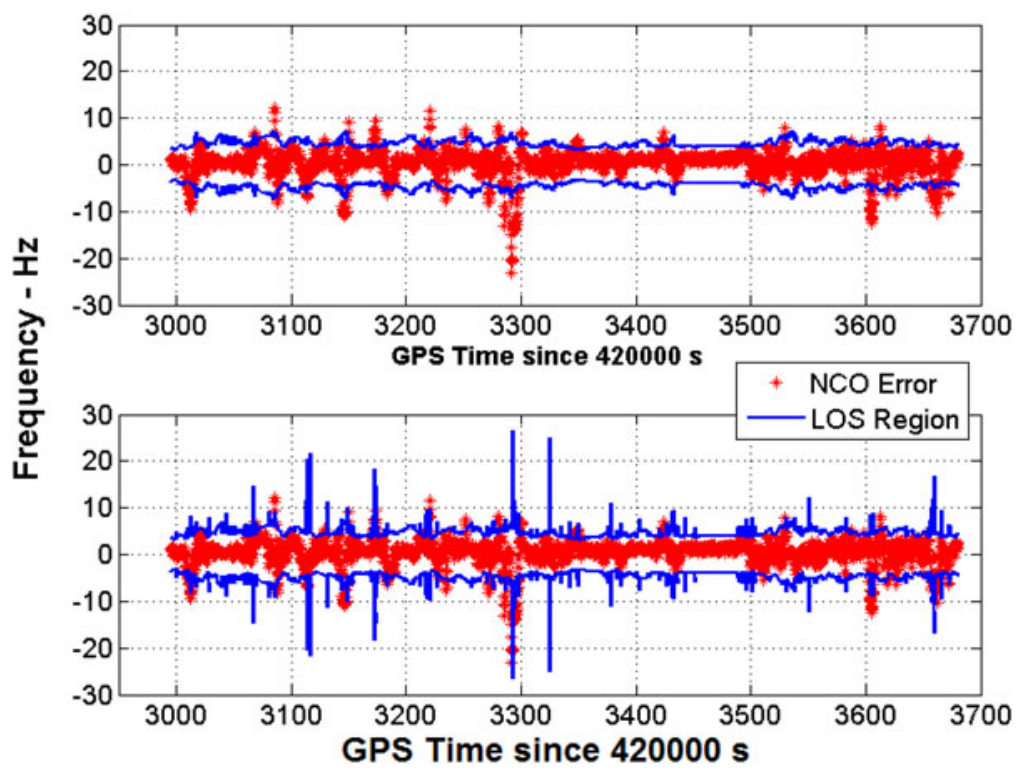

Figure 8. PRN 4 NCO errors and associated receiver LOS region before (upper figure) and after (lower figure) the receiver anomaly check.

Figure 8 shows the NCO errors and the predicted LOS regions of PRN 4 in the Doppler domain before (top) and after (bottom) anomaly detection. First, it is observed that these are smaller than with the dominant peak strategy (see Figure 5). This demonstrates the algorithm's ability to mitigate the effect of NLOS signals at the measurement level. Second, the NCO errors are generally smaller than the LOS region, even before the anomaly detection algorithm is applied. This suggests that the variance-covariance matrix from the Kalman filter is a reasonable approximation of the velocity accuracy. That said, there are a few instances where the NCO error falls outside of the LOS region. This can be observed, for example, at the time $423293.6 \mathrm{~s}$ where the frequency errors reach about $24 \mathrm{~Hz}$, which corresponds to when the North velocity error reaches $6 \mathrm{~m} / \mathrm{s}$ (Figure 7). Because the peak falls outside the LOS region if the anomaly detection is not applied, the peak would be excluded from the receiver. However, the re-scaled version of the LOS region (after the anomaly detection algorithm) is shown in the lower part in Figure 8. Notice how the number of times the NCO error falls outside the LOS region has been dramatically reduced.

Figure 9 shows a zoomed-in version during the period of $423170 \mathrm{~s}$ to $423180 \mathrm{~s}$ where it is observed that after receiver anomaly detection, the LOS signals (if present) can be observed in the LOS region (i.e., NCO frequency errors are smaller than the LOS region).

The NCO errors in the code phase domain are not shown because the receiver cannot detect any receiver anomaly in the code phase/position domain. Table 5 summarises the number of anomalies on each satellite before and after receiver anomaly detection is applied. Here, the occurrence of an anomaly is determined by comparing the receiver NCO values with the reference values in post processing. In other words, 


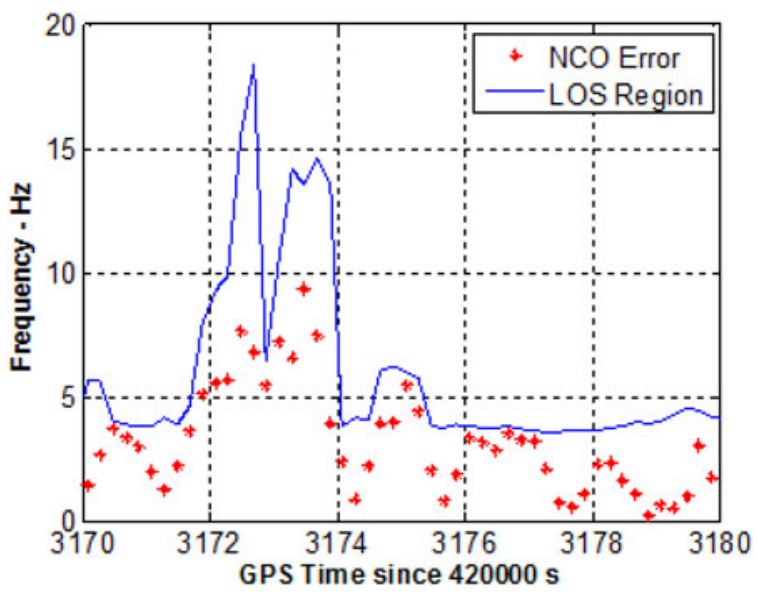

Figure 9. PRN 4 NCO frequency errors and associated receiver LOS region after the receiver anomaly check zoomed-in.

Table 5. Receiver Anomalies before and after the Receiver Anomaly Detection.

\begin{tabular}{lccccrc}
\hline & \multicolumn{2}{l}{$\begin{array}{l}\text { Velocity } \\
\text { (Frequency) } \\
\text { Anomalies }\end{array}$} & & & \multicolumn{2}{l}{$\begin{array}{l}\text { Position (Code } \\
\text { Phase) Anomalies }\end{array}$} \\
\cline { 2 - 3 } PRN & Before & After & Impro-vement & Before & After & Impro-vement \\
\hline 2 & 227 & 112 & $51 \%$ & 53 & 53 & $0 \%$ \\
4 & 97 & 43 & $54 \%$ & 0 & 0 & $0 \%$ \\
9 & 61 & 26 & $57 \%$ & 0 & 0 & $0 \%$ \\
12 & 264 & 192 & $27 \%$ & 263 & 263 & $0 \%$ \\
17 & 80 & 42 & $48 \%$ & 0 & 0 & $0 \%$ \\
27 & 53 & 15 & $72 \%$ & 0 & 0 & $0 \%$ \\
28 & 233 & 142 & $39 \%$ & 14 & 14 & $0 \%$ \\
\hline
\end{tabular}

these are not the anomalies reported by the receiver itself (note that a total of 3547 epochs were processed).

Before anomaly detection, NCO frequency/velocity anomalies occur at more than 200 epochs for PRN 2, PRN 12, and PRN 28. In contrast, anomalies occur at fewer than 100 epochs for the rest of the constellation. This is because a velocity error manifests itself differently for each satellite (based on their azimuth and elevation angles) thus making it easier or harder to detect. Also, from this table it is observed that NCO code phase anomalies occur less frequently than NCO frequency anomalies.

More importantly, after anomaly detection, the number of anomalies are reduced substantially. It is noted, however, that the receiver anomaly cannot be removed completely. No position anomaly has been reported in the receiver, thus no improvement is shown in this table in the code phase domain. Ultimately, this suggests that the frequency/velocity anomaly detection is more critical than the code phase/position anomaly detection. 


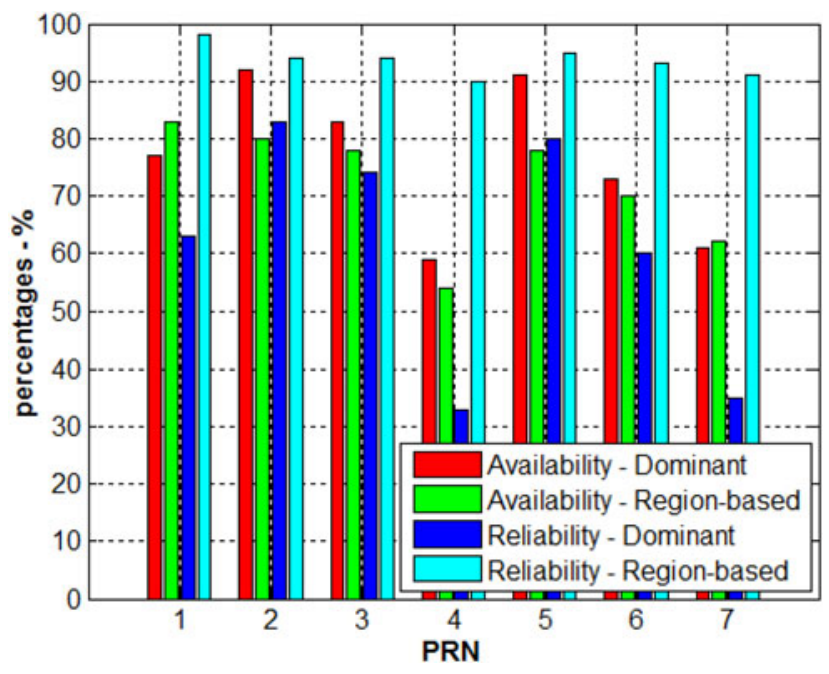

Figure 10. Dominant peak strategy and the region-based strategy availability and reliability comparison.

Figure 10 compares the dominant peak strategy performance and the region-based strategy performance in terms of availability and reliability percentages. Several conclusions can be drawn from this figure. First, the availability is reduced substantially for low elevation satellites compared to the dominant peak strategy (recall Table 3). Second, as expected, high elevation satellites generally have better availability than low elevation satellites. The availability is $83 \%$ for PRN 4 with an elevation of $50^{\circ}$, and $35 \%$ for PRN 28 with an elevation of $24^{\circ}$. Third, the reliability of the regionbased approach is high with at least $90 \%$ of the multipath peaks being removed.

5. CONCLUSIONS. This paper presented a peak selection method to improve position performance for high sensitivity receivers. Different receiver architectures are applied and compared, including a commercial high sensitivity receiver and three proposed high sensitivity receiver strategies, namely: a dominant peak strategy, a powerbased strategy, and a region-based strategy. The following conclusions can be drawn from the results presented:

- Large position and velocity variations are observed in the dominant peak strategy, this is due to NLOS signals with large pseudorange and/or Doppler errors being considered as the LOS peaks in the receiver.

- The proposed power-based strategy returns the worst positioning performance compared to other high sensitivity strategies. This is due to the receiver not being able to identify enough LOS signals in weak signal environments, thus reducing availability.

- The proposed region-based LOS peak strategy yields RMS horizontal position errors close to $20 \mathrm{~m}$ and the RMS horizontal velocity errors close to $0.6 \mathrm{~m} / \mathrm{s}$. It is shown that the reliability for a low elevation satellite is improved from 
around $60 \%$ to $90 \%$; and for a high elevation satellite, it is improved from around $75 \%$ to $95 \%$. Most of the multipath signals are removed from the receiver.

- Applying the anomaly detection algorithm reduces the number of undetected frequency errors by between $27 \%$ and $57 \%$, depending on the satellite.

\section{ACKNOWLEDGMENTS}

The financial support of General Motors of Canada and the Natural Science and Engineering Research Council of Canada is acknowledged.

\section{REFERENCES}

Borio, D., O'Driscoll, C. and Lachapelle, G. (2008). Coherent, Non-Coherent and Differentially Coherent Combining Techniques for the Acquisition of New Composite GNSS Signals. IEEE Transactions on Aerospace and Electronic Systems, 45(3), 1227-1240.

Gaggero, P. O. (2008). Effect of Oscillator Instability on GNSS Signal Integration Time, Master Thesis, Faculty of Science Institute of Microtechnology, University of Neuchatel.

Gaggero, P. O., and Borio, D. (2008). Ultra-stable Oscillators: Limits of GNSS Coherent Integration. Proceedings of the 21st International Technical Meeting of the Satellite Division of The Institute of Navigation, September, Savannah, GA, 565-575.

Gunawardena, S., van Graas, F. and Andrey, S. (2004). Real Time Block Processing Engine for Software GNSS Receivers, Proceedings of the 2004 National Technical Meeting of The Institute of Navigation, San Diego, CA, 371-377.

Kaplan, E. D. and Hegarty, C. (2006). Understanding GPS: Principle and Applications. Second Edition, Artech House, Boston.

Langley, R. B. (1997). GPS Receiver System Noise, GPS World, 8(6), 40-45.

Lashley, M. and Bevly, D.M. (2008). Comparison of Traditional Tracking Loops and Vector Based Tracking Loops for Weak GPS Signals. Proceedings of the 2008 National Technical Meeting of The Institute of Navigation, San Diego, CA, 310-316.

Lin, T., O'Driscoll, C. and Lachapelle, G. (2011), Development of a Context-Aware Vector-Based HighSensitivity GNSS Software Receiver. Proceedings of the 2011 International Technical Meeting of The Institute of Navigation, San Diego, CA, 1043-1055.

Misra, P. and Enge, P. (2006). Global Positioning System: Signals, Measurements and Performance, 2nd Edition, Ganga-Jamuna Press.

O'Driscoll, C., Lachapelle, G. and Tamazin, M. (2011). Dynamic Duo: Combined GPS/GLONASS Receivers in Urban Environments, GPS World, January 1, 51-58.

Parkinson, B. W. and Spilker, J.J. (1996). Global Positioning System: Theory and Applications. Vol.1, American Institute of Aeronautics and Astronautics publication, Inc.

Psiaki, M. L. (2001). Block Acquisition of Weak GPS Signals in a Software Receiver. Proceedings of the 14th International Technical Meeting of the Satellite Division of The Institute of Navigation (ION GPS 2001), Salt Lake City, UT, 2838-2850.

Soloviev, A. and van Graas, F. (2008). Utilizing Multipath Reflections in Deeply Integrated GPS/INS Architecture for Navigation in Urban Environments. Proceedings of ION/IEEE PLANS 2008, May 5-8, Monterey, CA, 383-393.

Van Graas, F., Soloviev, A., Uijt de Haag, M., Gunawardena, S., and Braasch, M. (2005). Comparison of Two Approaches for GNSS Receiver Algorithms: Batch Processing and Sequential Processing Considerations. Proceedings of the 18th International Technical Meeting of the Satellite Division of The Institute of Navigation, Long Beach, CA, 200-211.

Xie, P., Petovello, M. G., and Basnayake, C. (2011). Multipath Signal Assessment in the High Sensitivity Receivers for Vehicular Applications. Proceedings of the 24th International Technical Meeting of The Satellite Division of the Institute of Navigation, September 18-22, Portland, OR, 1764-1776. 
Xie, P. and Petovello, M. G. (2012). Improving High Sensitivity Receiver Performance in Multipath Environment for Vehicular Applications. Proceedings of the 25th International Technical Meeting of The Satellite Division of the Institute of Navigation, September 17-21, Nashville, TN, 448-458.

Xie, P. (2013). Improving High Sensitivity GNSS Receiver Performance in Multipath Environments for Vehicular Applications. PhD Thesis, Department of Geomatics Engineering, University of Calgary.

Xie, P. and Petovello, M.G. (2014). Measuring GNSS Multipath Distributions in Urban Canyon Environments. IEEE Transactions on Instrumentation and Measurement, 64(2), 366-377.

Zhang, J., Zhang, K., Grenfell, R., and Deakin, R. (2006). GPS Satellite Velocity and Acceleration Determination using the Broadcast Ephemeris. The Journal of Navigation, 59, 293-305. 